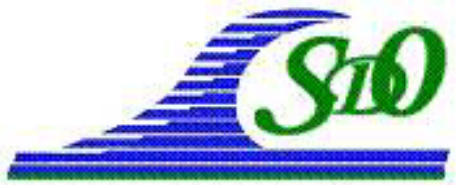

XI ìmes Journées Nationales Génie Côtier - Génie Civil

Les Sables d'Olonne, 22-25 juin 2010

DOI:10.5150/jngcgc.2010.019-A C Editions Paralia CFL

disponible en ligne - http://www.paralia.fr - available online

\title{
Répartition des minéraux entre Ras Taguermess et Ras Marmour (Golfe de Gabès) : Relation avec la dynamique côtière
}

\author{
Abdelfattah ATOUI ${ }^{1}$, Mouldi BRAHIM ${ }^{1}$ \\ 1. Institut National des Sciences et Technologies de la Mer, 28 rue 2 mars 1934, \\ Salambô, 2025 Tunis, Tunisie. \\ abdelfattah.atoui@gmail.com ; mouldi.brahim@instm.rnrt.tn
}

\section{Résumé :}

La distribution spatiale des minéraux dans les sédiments des petits fonds de la frange littorale orientale de Djerba dans le golfe de Gabès donne un aperçu sur la dynamique sédimentaire de la zone. Le quartz est un bon traceur qui permet de déterminer le sens de la dérive littorale, qui est ici générée par les vagues sous le contrôle des vents de direction Est à Nord-Est. Il est plus abondant au nord entre Ras Taguermess et Sidi Garous où les plages sont relativement importantes; il diminue le long de la zone érodée entre Sidi Garous et Aghir. Le transport s'effectue donc du nord vers le sud et on observe une concentration assez importante de ce minéral dans la région de Bin El Ouediane.

La distribution spatiale des carbonates de calcium et de la calcite magnésienne montre que cette frange littorale est alimentée aussi par des apports des bassins versants limitrophes. Les sédiments des petits fonds sont relativement riches en débris de coquilliers.

\section{Mots clés :}

Minéraux - Dynamique sédimentaire - Djerba - Golfe de Gabès

\begin{abstract}
:
The spatial distribution of minerals in the sediment of the small funds of the oriental littoral fringe of Djerba in the gulf of Gabes looked an outline onto the sedimentary dynamics of the zone. The quartz is a good tracer who gave the sense of the littoral drift to generate by waves creates by the winds of direction of the East to Northeast. It is more plentiful in the North between Ras Taguermess and Sidi Garous where beaches are relatively important and declines in the zone affected between Sidi Garous and Aghir. The transport has a direction of the North to the South and we have an important sedimentation in the region of Bin El Ouediane.

The spatial distribution of the carbonates of calcium and the magnesium calcite shows that this littoral fringe is fed by land transport sediments resulting from ponds bordering hillsides. Sediments of the small funds are relatively rich in coquilles fragment.
\end{abstract}

Key words:

Minerals - Sediment dynamics - Djerba - Gulf of Gabes 


\section{Introduction}

La zone d'étude est située au sud-est de la Tunisie. Elle est située à l'est et au sud-est de Djerba entre $33^{\circ} 35^{\prime}$ et $33^{\circ} 50^{\prime}$ de latitude nord et $10^{\circ} 55^{\prime}$ et $11^{\circ} 05^{\prime}$ de longitude est (figure 1).

Située entre les caps Ras Taguermess-Ras Marmour, cette partie de la frange littorale est soumise à une dégradation et à une perturbation de son budget sédimentaire (PASKOFF, 2004 ; OUESLATI, 2004 ; MASMOUDI et al., 2005). Ce déséquilibre sédimentaire est la résultante des effets naturels (vents, houles et marée), des actions anthropiques et de déficits en apports terrigènes par les cours d'eaux exoréiques (MIOSSEC \& PASKOFF, 1979 ; BOURGOU, 1993 ; OUESLATI, 2004).

Cette zone fait partie de l'étage aride. Elle est structurée par des plis de direction nordouest, à l'origine d'affleurements quaternaires: Villafranchien, Tyrrhénien, Néotyrrhénien et Flandrien (BOUAZIZ et al., 2003). Les surfaces continentales limitrophes sont formées essentiellement de sols calcimorphes, de sols isohumiques, de sols salés et de sols peu évolués, riches en encroûtements. Ceux-ci sont soit calcaires d'âge villafranchiennes et résistants, soit gypseuses, alors sensibles à l'érosion hydrique et à l'érosion éolienne (BOUAZIZ et al., 2003 ; OUESLATI, 2004).

Le transport solide est acheminé vers la lagune de Boughrara par les principaux oueds (Bou Hamed, El Fjé et Smar,). Il est de l'ordre $11 \mathrm{Mm}^{3} /$ an (DGPA, 2000).

De nombreux secteurs côtiers de l'île de Djerba connaissent un recul plus ou moins important de la ligne de rivage. Ce recul concerne particulièrement les côtes exposées aux vents des secteurs nord/nord-est et est et notamment entre Ras Taguermess et Ras Marmour. Les vents dominants et les plus intenses sont du secteur est à nord/nord-est (INM, 2008)

La présente étude a pour but d'interpréter la dynamique des sédiments superficiels de la frange littorale située entre Ras Taguermess et Ras Marmour par le biais d'une étude minéralogique.

\section{Matériels et méthodes}

Trois campagnes d'échantillonnage des sédiments de surface ont été effectuées durant les mois de mai et juillet 2008. Elles couvrent l'ensemble de la zone d'étude (figure 1). Pendant ces campagnes, nous avons prélevés 28 échantillons des sédiments de la surface du petit fond entre 0 et $10 \mathrm{~m}$ de profondeur à l'aide d'une benne de type Van Veen. Les positions ont été déterminées au GPS.

Les échantillonnages des sédiments de surface, couvrant l'ensemble de la zone entre Ras Taguermess et Ras Marmour, ont été l'objet d'une analyse minéralogique.

Les analyses minéralogiques consistent à déterminer les minéraux argileux et non argileux par diffraction aux rayons $\mathrm{X}$. 


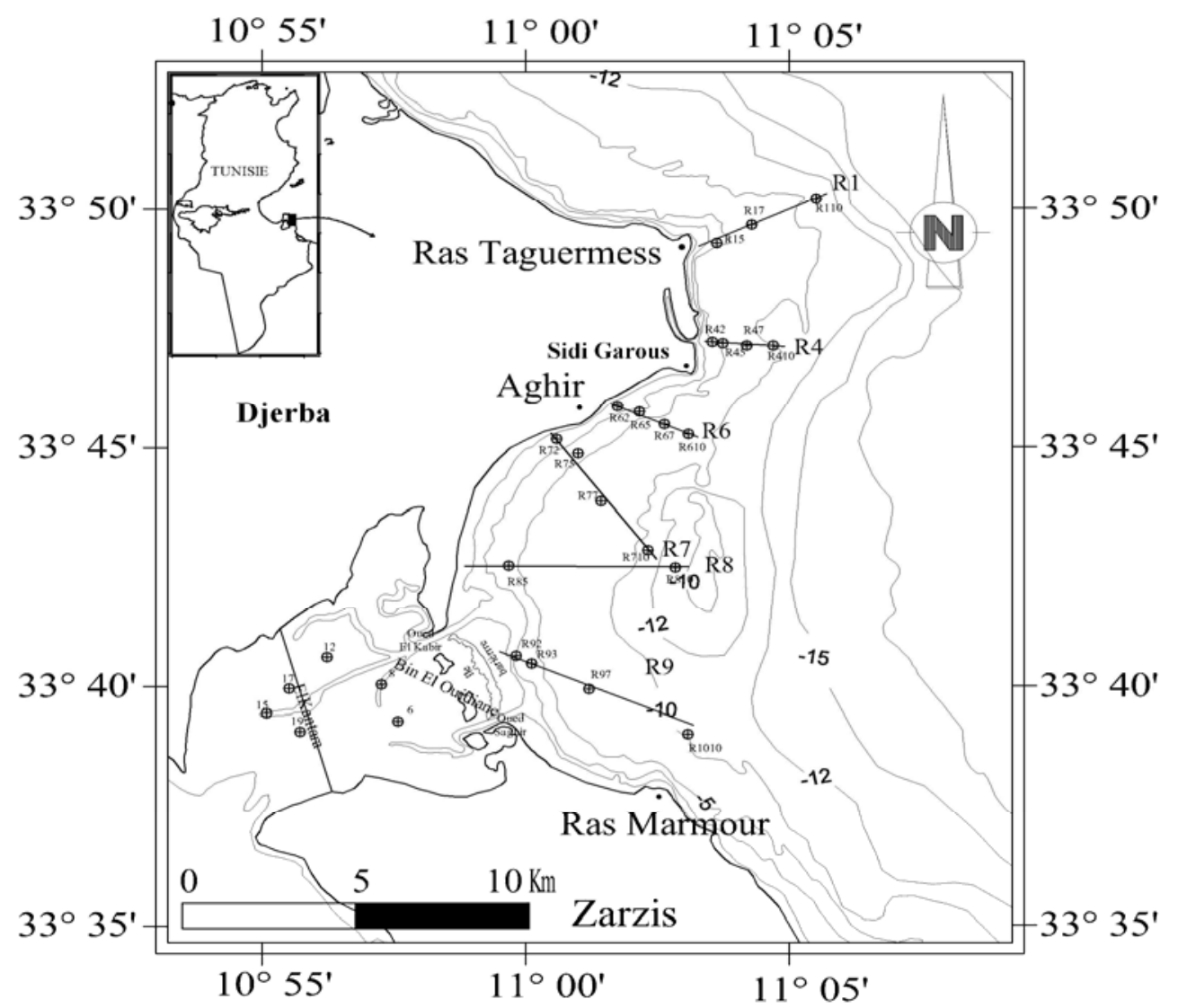

Figure 1. Localisation des stations de prélèvements des sédiments de surface (mai-juillet 2008).

\section{Résultats et interprétation}

\subsection{Minéralogie du sédiment total}

Les résultats de diffraction aux rayons $\mathrm{X}$ des échantillons totaux montrent la présence des minéraux suivants selon leur ordre de pourcentage relatif : quartz, aragonite, calcite magnésienne, calcite et dolomite (figure 2).

Par la méthode semi-quantitative, nous avons établi la carte de la distribution spatiale des minéraux non argileux (figure 2).

\section{a) Quartz}

Le quartz $\left(\mathrm{SiO}_{2}\right)$ est le minéral le plus fréquent (41\%). Sa distribution spatiale (figure 3) montre que les valeurs de pourcentage sont plus importantes entre Ras Taguermess et Aghir. Le pourcentage reste élevé en longeant l'isobathe $-5 \mathrm{~m}$ vers le Sud dans la baie d'Aghir. Sa teneur diminue, généralement, en allant de la côte vers le large. L'existence du quartz est due à l'érosion des formations géologiques bordières limitrophes (BOUAZIZ et al., 2003). Il peut être transporté par voie hydrique ou éolienne. 
La figure 3 montre que les grains de quartz se déplacent du nord vers le sud selon la direction des courants de dérive littorale. En effet, la houle de direction Est à Nord-Est crée des courants de dérive le long de la frange littorale entre Ras Taguermess et le cap de Sidi Garous et un courant de retour qui est responsable du transport des sédiments fins vers le large.

Le courant de dérive qui est généré par les houles se scinde en deux parties. Une partie longe la frange littorale jusqu'à Aghir. L'autre partie prend la direction du nord vers le sud jusqu'à l'île barrière de Bin El Ouediane. Ces deux courants sont responsables du transport des sables (figure 3). La diminution du stock sableux entre Ras Taguermess et Aghir indique une érosion assez importante dans cette zone (MIOSSEC \& PASKOFF, 1979 ; OUESLATI, 2004).

En effet, en nous fondant sur les études antérieures sur la dynamique des eaux (SAMMARI et al., 2006; ALIOUA \& HARZALLAH, 2008) et sur les études sur la dynamique sédimentaire (BEN AMOR et al., 2003; ROBERGE \& LONG, 2003 ; MASMOUDI et al., 2005 ; ATOUI \& BRAHIM, 2009) nous avons établie sur une carte (figure 4) de la circulation et des courants de la dérive dans la frange littorale entre Ras Taguermess et Ras Marmour).

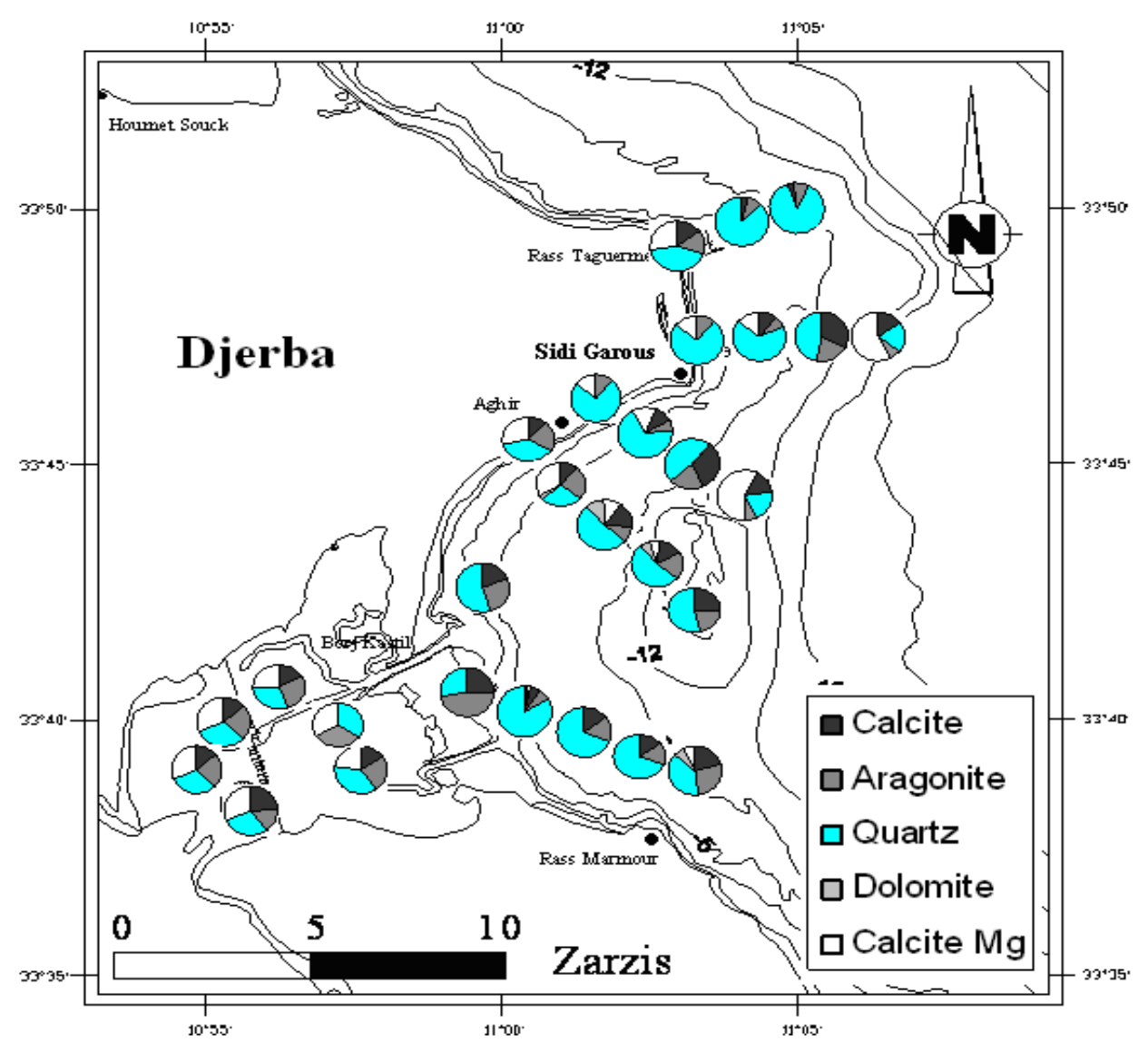

Figure 2. Distribution spatiale des minéraux non argileux. 


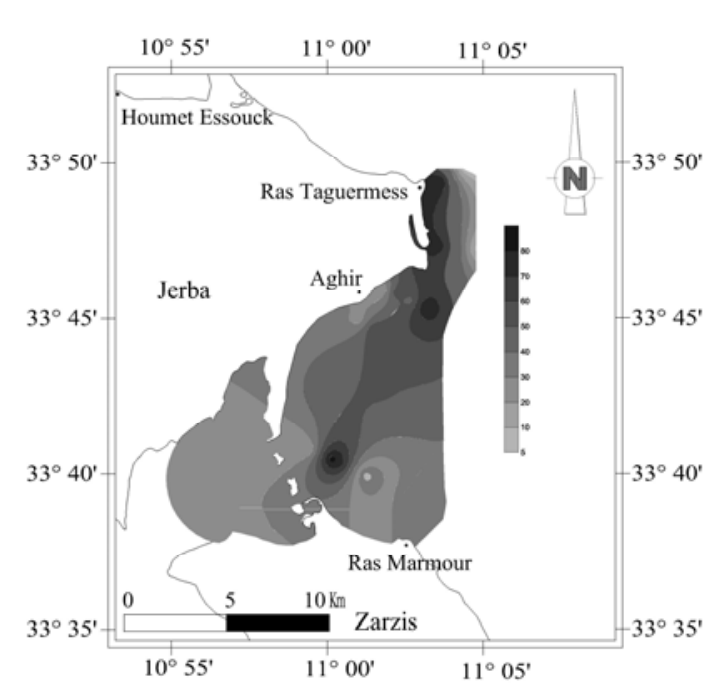

Figure 3. Distribution spatiale du quartz.

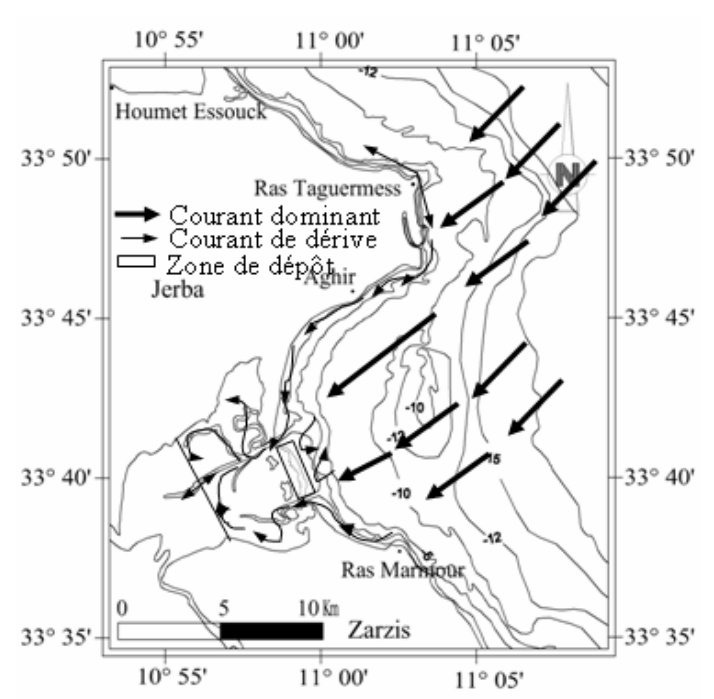

Figure 4. Schéma synthétique des circulations dominantes d'eau dans la zone d'étude.

La distribution spatiale du quartz a permis de conclure que la zone entre Ras Taguermess et Aghir est une zone d'érosion et la zone centrale de la baie au niveau de Bin El Ouediane est une zone de dépôt.

\section{b) Le carbonate de calcium $\mathrm{CaCO} 3$}

Le carbonate de calcium ( $\mathrm{CaCO} 3)$ existe sous deux formes cristallines dans les échantillons : la calcite et l'aragonite.

Dans l'ensemble des échantillons de la zone d'étude l'aragonite varie entre $5 \%$ et $36 \%$ (figure 5). Les teneurs les plus importantes sont localisées dans les petits fonds, notamment dans la zone de Bin El Ouediane et au niveau de Ras Marmour, zone qui est riche en débris coquilliers

La calcite varie entre $0 \%$ et $29 \%$ dans les échantillons prélevés sur les petits fonds entre Ras Taguermess et Ras Marmour (figure 6). Elle devient généralement de plus en plus fréquente de la côte vers le large. La teneur en calcite est, particulièrement, plus importante en face des caps $(29 \%)$ vu la richesse de la zone en débris coquilliers. Mais il faut tenir compte aussi de la présence d'apports détritiques issus du continent.

\section{c) La calcite magnésienne}

Les teneurs de la calcite magnésienne varient entre $0 \%$ et $55 \%$ (figure 7 ). Les teneurs les plus importantes sont localisées près des côtes et en face du cap de Ras Taguermess. D'après les travaux de BEN AMOR (2007) dans le golfe de Gabès, ce minéral a été observé au cours de la saison estivale avec un rapport $\mathrm{Mg} / \mathrm{Ca}>2,8 \mathrm{~mol}^{-1}$. Toujours d'après cet auteur, il serait d'origine biogénique. 


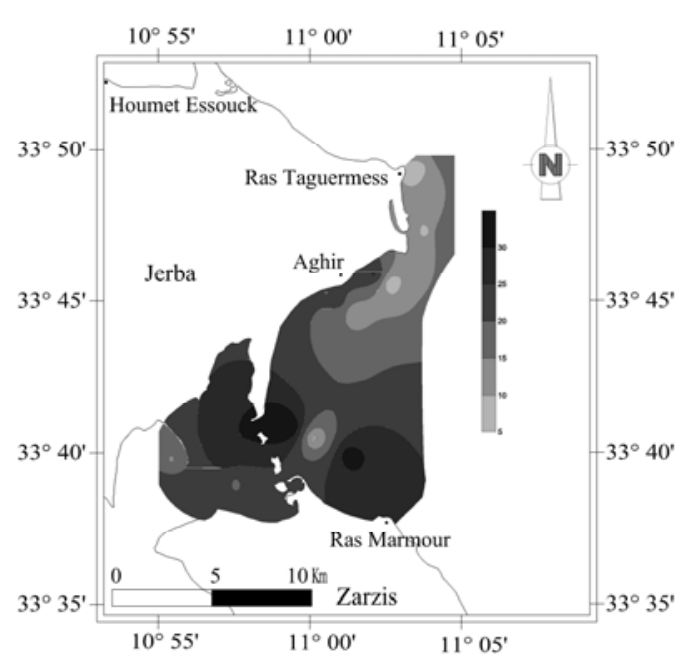

Figure 5. Distribution spatiale de l'aragonite.

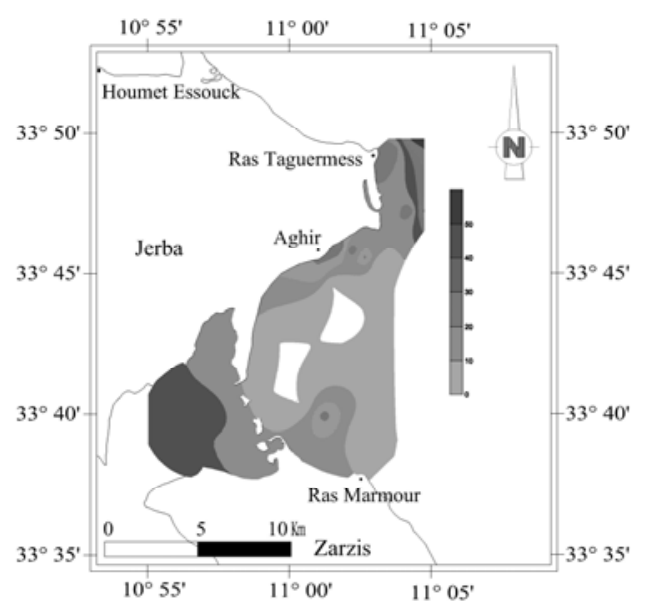

Figure 7. Distribution spatiale de la calcite magnésienne.

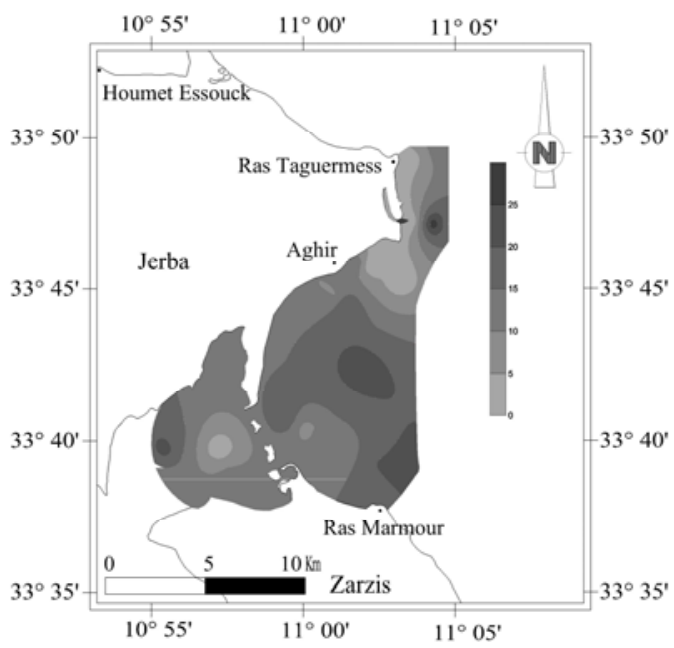

Figure 6. Distribution spatiale de la calcite.

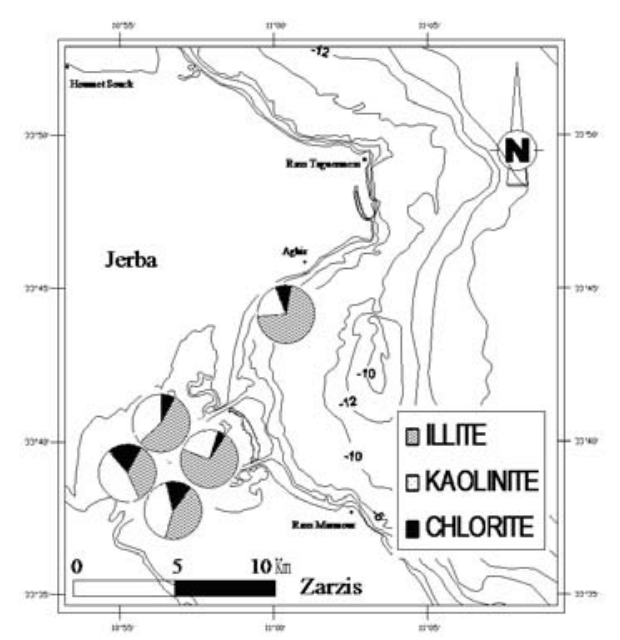

Figure 8. Pourcentage des minéraux argileux dans la zone d'étude.

\subsection{Minéralogie des argiles}

Les argiles sont localisées essentiellement dans la zone à faible circulation dans la région de Bin El Ouediane. Les minéraux argileux identifiés sont la kaolinite, l'illite et la chlorite (figure 8).

- L'illite est le minéral argileux le plus abondant, avec des teneurs comprises entre $43,77 \%$ et $70,62 \%$ (figure 8 ).

- La kaolinite est présente avec des teneurs allant de $20,47 \%$ à $41,73 \%$. Elle est transporté par les courants de marée (MARAI, 2005) et se sédimente dès que les courants sont faibles près d'El Kantara.

- Les teneurs en chlorite, probablement néoformée, varient entre 8,91\% et 14,45\% dans les sédiments superficiels entre Ras Taguermess et Ras Marmour. 


\section{Conclusion}

L'étude minéralogique des sables prélevés de la frange orientale de Djerba entre 0 et 10 $\mathrm{m}$ de profondeur montre que ces sables sont constitués de quartz, d'aragonite, de calcite magnésienne, de calcite et de dolomite.

Le quartz est le minéral le plus abondant. A travers l'évolution spatiale, de son abondance relative nous avons pu définir le sens de la dérive littorale et des courants de retour créés par la houle d'Est à Nord-Est.

L'aragonite et la calcite ont deux origines. Elles sont apportées par les cours d'eaux en période de crue, provenant alors des calcrètes (croûte calcaire pédologiques) d'age Villafranchien. Il s'agit aussi de débris coquilliers. La calcite magnésienne des échantillons prélevés provient d'une néoformation et/ou d'un héritage des bassins versants limitrophes. La dolomite se présente en très faibles pourcentages dans les échantillons prélevés dans les profondeurs de $10 \mathrm{~m}$. Elle serait néoformée.

Les résultats de l'analyse des minéraux argileux montrent que les argiles sont constituées de l'illite, de la kaolinite et de chlorite. La kaolinite et l'illite sont d'origine terrigène, transportées par les cours d'eaux limitrophes de la région de Boughrara. Par contre la chlorite serait néoformée en milieu marin.

\section{Références bibliographiques}

ALIOUA M., HARZALLAH A. (2008). Imbrication d'un modèle de circulation des eaux prés des côtes tunisiennes dans un modèle de circulation de la Méditerranée. Bull. Inst. Natn. Scien. Tech. Mer de Salambô, Vol 35, Tunisie, pp 169-176.

ATOUI A., BRAHIM M. (2009). Distribution granulométriques des sédiments de surface de la frange littorale du Ras Taguermess au Ras Marmour (Golfe de Gabès, Tunisie). Conférence Méditerranéenne Côtière et Maritime, Edition 1, Hammamet, Tunisie, pp 75-78. doi:10.5150/cmcm.2009.019

BEN AMOR R. (2007). Géochimie des eaux et des sédiments du littoral GhannoucheGabès (Golfe de Gabès), Impact des rejets de phosphogypse. Thèse en géologie. FST, $189 \mathrm{p}$.

BEN AMOR R., BRAHIM M., GUEDDARI M. (2003). Essai d'interprétation de la dynamique sédimentaire par l'analyse granulométrique et minéralogique au large du golfe de Gabès. Bull. Inst. Natn. Scien. Tech. Mer de Salammbô, Vol. 30, Tunisie, pp 143-151

BOUAZIZ S., JEDOUI Y., BARRIER E., ANGELIER J. (2003). Néotectonique affectant les dépôts marins tyrrhéniens du littoral sud-est tunisien : implications pour les variations $d u$ niveau marin. C. R. Géoscience 335, pp 247-254. doi:10.1016/S1631-0713(03)00031-2

BOURGOU M. (1993). L'impact des mutations socio-économiques récentes sur le milieu naturel à Djerba, le cas de littoral nord-oriental. Revue Tunisienne de Géographie, 23-34, pp 69-86. 
Thème 2 -Dynamique sédimentaire et transports des particules

DGPA -Direction Générale de la Pêche et de l'Aquaculture- (2000). Etude de l'amélioration de la qualité de l'eau de la lagune de Boughrara, Phase I-Partie II, Diagnostic de la situation actuelle. SCET-TUNISIE-ERI, Ch IV, $326 \mathrm{p}$.

INM (2008). Données climatiques et statistique annuelles des vents (1981-2004).

MARAI G. (2005). Etude qualitative et quantitative de la marée dans le golfe de Gabès, Mastère en hydrodynamique et modélisation de l'environnement. Ecole Nationale des Ingénieurs de Tunis, $87 \mathrm{p}$.

MASMOUDI S., YAICH C., YAMOUN M. (2005). Evolution et morphodynamique des îles barrières et des flèches littorales associées à des embouchures microtidales dans le Sud-Est tunisien. Bulletin de l'institue Scientifique, section Sciences de la Terre, pp 65-81.

MIOSSEC J.-M., PASKOFF R. (1979). L'érosion des plages dans le sud tunisien: exemples à Djerba (côte est et sud-est) et à Zarzis. Revue Tunisienne de Géographie, 2, pp 137-155.

OUESLATI A. (2004). Littoral et aménagement en Tunisie. Des enseignements de l'expérience du vingtième siècle et de l'approche géoarchéologique à l'enquête prospective. Faculté des Sciences humaines et Sociales Tunis. 534 p.

PASKOFF R. (2004). L'érosion des côtes: le cas des plages de l'île de Djerba (Tunisie), La Houille Blanche, $\mathrm{n}^{\circ} 1$.

ROBERGE C., LONG B. (2003). Etude de l'estimation de transport sédimentaire sur le littoral Nord-Est de Djerba, Tunisie. Conférence canadienne sur le littorale, $17 \mathrm{p}$.

SAMMARI C., KOUTITONSKY V.G., MOUSSA M. (2006). Sea level variability and tidal resonance in the Gulf of Gabes, Tunisia. Continental Shelf Research, 26, pp 338-350. doi:10.1016/j.csr.2005.11.006 